\title{
G

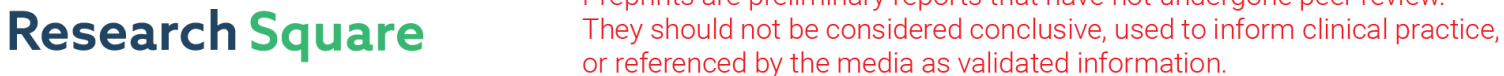

\section{Increased Tea Saponin Content Influences The Diversity and Function of Plantation Soil Microbiomes}

\section{Shouke Zhang}

Chinese Academy of Forestry https://orcid.org/0000-0002-7941-2029

Junqia Kong

Chinese Academy of Science

\section{Zikun Li}

Zhejiang Agriculture and Forestry University: Zhejiang A and F University

\section{Feng Song}

Zhejiang $A$ and $F$ University

\section{Xinhua He}

Zhoushan Academy of Forestry Science

\section{Longfei Chen}

Chinese Academy of Sciences

\section{Kai Guo ( $\nabla$ kaiguo@zafu.edu.cn )}

Zhejiang Agriculture and Forestry University: Zhejiang A and F University

\section{Xudong Zhou}

Zhejiang Agriculture and Forestry University: Zhejiang A and F University

\section{Research}

Keywords: Plant secondary metabolites, soil microbiome, plantation ages, soil physicochemical properties, core function

Posted Date: November 1st, 2021

DOI: https://doi.org/10.21203/rs.3.rs-1004193/v1

License: (c) (i) This work is licensed under a Creative Commons Attribution 4.0 International License. Read Full License

Version of Record: A version of this preprint was published at Microbiology Spectrum on January 12th, 2022. See the published version at https://doi.org/10.1128/spectrum.02324-21. 


\section{Abstract \\ Background}

Plant secondary metabolites (PSMs) can affect the structures and functions of soil microbiomes. However, the core bacteria associated with PSMs, and their corresponding functions have not been explored extensively. In this study, soil physicochemical properties, tea saponin contents, microbial community compositions, and microbial community functions of different-age Camellia oleifera plantation soils from representative regions were analyzed. We evaluated the effects of plantation age increase on PSM accumulation, and the subsequent consequences on the structures and functions of soil microbiomes.

\section{Results}

Plantation ages increase positively corresponded with accumulated tea saponin contents, with negative effects on soil physicochemical properties, and soil microbiome structures and functions. Older plantation soil microbiomes exhibited simpler structures, lower diversity, and relatively looser putative interactions based on network analysis. Clearly, the core functions of soil microbiomes transitioned to those associated with PSM metabolisms, while microbial pathways involved in cellulose degradation were inhibited. Degradation experiments further confirmed that older plantation soils exhibited the higher capacity on tea saponin degradation but poorer on furfural.

\section{Conclusions}

This study systematically explored the influences of PSMs on soil microbiomes via the investigation of key bacterial populations and their functional pathways. With the increase of planting years, increased tea saponin content simplified the soil microbiomes diversity, inhibited the degradation of organic matter, and enriched the genes related to the degradation of tea saponin. These findings significantly advance our understanding on PSMs-microbiome interactions and could provide fundamental and important data for sustainable management of Camellia plantations.

\section{Background}

Artificial management leads to specific plants becoming the dominant species within a particular area and their litter can significantly impact soil characteristics including soil microbial composition and function [1-2]. Plant litter decomposition is a complex, long-term process [2-4]. In addition to the release of organic matter and nutrients, plant secondary metabolites (PSMs) that are recalcitrant to degradation are also released into soils [3]. PSMs play important roles in ecosystem processes like plant succession or litter decomposition by regulating the interactions between plants and soil microorganisms [3]. Healthy and stable soil microbiomes with high diversity can promote soil decomposition, providing an appropriate 
environment ideal for plant growth. However, most PSMs also have bacteriostatic effects that lead to degradation of critical soil physicochemical properties [5].

Many studies indicated that microbiomes are influenced by numerous types of molecules, including coumarins, glucosinolates, benzoxazines, and triterpenoids [1, 6-9]. These PSMs can exert a wide spectrum of effects upon individual microbial strains or groups, and particularly when they function as toxins [1]. Consequently, microbiome responses to PSMs primarily result in changes of microbial structures [10-14], and their functions accordingly [1, 13-14]. They include circulation of soil carbon, nitrogen, phosphorus, and other substances that directly affect plant growth and development and are closely related to plant health [4]. The plant growth decrease thus occurs mainly due to continuous planting of a single species over many years at large area and degradation of the circulation. For example, dominant plants have more significant effects on soil abiotic and biotic properties [15-16] that in turn influence litter decomposition via changes in decomposer communities. Previous studies solely evaluated the relationship between soil microbiome change, soil physicochemical property, and plant health $[1,13-14]$. The core bacteria associated with PSMs, and their functions thus deserve further investigation.

Camellia oleifera considers as an economically important woody edible oil crop and over 4 million ha plantation has been established in the mountainous areas of southern China [17]. The industry plays a critical role on poverty alleviation and direct economic output is worth approximately USD 6.5 billion annually [18]. However, Camellia plantation management with limited knowledge-based human intervention has led to weakened older Camellia plantations that are much less productive and hardly profitable $[17,19-21]$. Most studies attributed the results because of poor resistance of older $C$. oleifera to diseases and insects $[17,19-21]$, although soil compaction and high densities have also been investigated. Camellia plantations are generally established on hillsides and insufficient management has led to less soil compaction due to excessive fertilization [17, 21]. Therefore, it is possible that other factors influence the deterioration of older $C$. oleifera plantation conditions. Camellia oleifera tissues are rich in the triterpenoid saponin up to $10-20 \%$, which is a tea saponin composed of sugar chains and triterpenoids in addition to steroids or steroid alkaloids linked by carbon-oxygen bonds, and they are important PSMs involved in the disease and pest resistance [17, 21-22]. With the increase of planting years, $C$. oleifera litters are bound to accumulate in the soil, and considerable PSMs mainly of tea saponin may also decompose into the soil. Consequently, we propose the hypothesis that tea saponin affects the composition and function of soil microbiomes in older $C$. oleifera plantations, thereby influencing overall soil ecological functions.

To explore this hypothesis, soil samples were collected from three major oil tea producing areas in China that represent different plantation ages from different geographic regions. The relationships between soil physicochemical properties, plantation ages, and tea saponin accumulation levels were analyzed. In addition, 16S rRNA gene high-throughput sequencing and soil metagenomic sequencing were performed to analyze the effects of planting period and tea saponin content on soil microbiome structures and 
functions, respectively. Our results provide important insights into the reconstruction of low-productive $C$. oleifera plantations, and a framework for the analysis and management improvement.

\section{Materials And Methods}

\section{Study sites and sampling}

In this study, Qiangtian ( $\left.28^{\circ} 11^{\prime} 51.61^{\prime \prime} \mathrm{N}, 120^{\circ} 23^{\prime} 15.25^{\prime \prime} \mathrm{E}\right)$, Quzhou $\left(29^{\circ} 3^{\prime} 48^{\prime \prime} \mathrm{N}, 118^{\circ} 36^{\prime} 15^{\prime \prime} \mathrm{E}\right)$, and Jiande $\left(29^{\circ} 01^{\prime} 32.06^{\prime \prime} \mathrm{N}, 119^{\circ} 37^{\prime} 28.45^{\prime \prime} \mathrm{E}\right)$ were selected as sample collection sites within Zhejiang, China. The sites exhibit a subtropical monsoon climate and are all less than $300 \mathrm{~m}$ above sea level. Each site comprises a pure $C$. oleifera plantation and no chemical spray has taken place toto control diseases, insects, and weeds. Medicinal plants including Dicranopteris pedata, Polygonum perfoliatum, Cynodon dactylon, and Imperata cylindrica are also distributed throughout the sites. The annual average temperature there is $15-18^{\circ} \mathrm{C}$, the coldest in January of $3-9^{\circ} \mathrm{C}$, and the hottest in July $26-29^{\circ} \mathrm{C}$.

Precipitation is abundant with an annual average of $1,600 \mathrm{~mm}$. The clay content of the topsoil layer is $31.28 \%$ and the weathering degree of the soil minerals is high, with the powdery clay ratio ranging between 0.83 and 0.98 . The clay minerals in the soil are primarily kaolinite. The red soils are acidic, with a surface $\mathrm{pH}<5.5$.

At each site, soil samples were collected from a test plot where trees have been planted for $1,3,5,7$, and 9 years. Eighteen sampling points were randomly selected within plots for each planting year. Within the vertical ground projection of the canopy $(1.5 \mathrm{~m} \times 1.5 \mathrm{~m})$, five points were randomly selected for sampling in June 2020. The top litter layer was removed from the soil and a soil collector was wiped with the soil that was to be collected prior to sampling. The $0-20 \mathrm{~cm}$ soil layer was collected from each point and the soils collected for each aged plantation were randomly divided into six samples. A total of 90 soil samples were collected using the same method from three sites. Fresh soils were sorted by hand to remove roots and stones. All samples were further divided into two sets and placed in sterile bags. The first soil portion was immediately frozen in liquid nitrogen, stored on dry ice, and transported back to the laboratory, followed by storage at $-80^{\circ} \mathrm{C}$ until subsequent DNA extraction. The other set was passed through a $2 \mathrm{~mm}$ sieve and stored at $4^{\circ} \mathrm{C}$ for determination of soil physicochemical properties.

\section{Soil physicochemical properties}

Soil physicochemical properties including $\mathrm{pH}$, moisture content (SW, \%), density $\left(\mathrm{SD}, \mathrm{g} \cdot \mathrm{cm}^{-3}\right)$, organic matter (SOM, $\left.\mathrm{mg} \cdot \mathrm{g}^{-1}\right)$, total nitrogen $\left(\mathrm{TN}, \mathrm{mg} \cdot \mathrm{g}^{-1}\right)$, total phosphorus $\left(\mathrm{TP}, \mathrm{mg} \cdot \mathrm{g}^{-1}\right)$, available phosphorus $\left(\mathrm{AP}, \mathrm{mg} \cdot \mathrm{kg}^{-1}\right)$, total potassium $\left(\mathrm{TK}, \mathrm{mg} \cdot \mathrm{g}^{-1}\right)$, and available potassium $\left(\mathrm{AK}, \mathrm{mg} \cdot \mathrm{kg}^{-1}\right.$ ) were determined as described by Jiang et al. (2021). Tea saponin (TS, $\mathrm{mg} \cdot \mathrm{g}^{-1}$ ) contents were determined with liquid chromatography [21] (Zhang et al., 2020). Correlational analysis of all soil physicochemical properties and planting years was conducted using the R software package (V. 4.0) [21] (Zhang et al., 2020).

\section{DNA extraction and high-throughput sequencing}


Bacterial DNA was extracted using a Qiagen DNeasy Blood and Tissue kit, followed by determination of DNA concentrations and size distributions. The extracted DNA from each sample was used as input for library preparation using the TruSeq Nano DNA LT Library Prep kit. Prior to sequencing, an Agilent BioAnalyzer was used to evaluate library quality using an Agilent High Sensitivity DNA kit. After validating the libraries, they were quantified using a Quant-iT PicoGreen dsDNA Assay kit from Promega Quant fluor. Polymerase chain reaction (PCR) was used to amplify the V5 - V7 hypervariable regions of bacterial 16S rRNA genes. 16S rRNA gene amplification was performed using the forward primer 799F (5'-AACMGGATTAGATACCCKG-3') and the reverse primer 1193R (5'-ACGTCATCCC-CACCTTCC-3') [23] (Jiang et al., 2021). Paired-end sequencing $(2 \times 300 \mathrm{bp})$ of quality-validated samples was conducted on the Illumina Miseq platform using the MiSeq Reagent kit V3 (600 cycles) [23] (Jiang et al., 2021). A target fragment size of $200-450$ bp was used for library construction that was performed at the Personal Biotechnology Company, Shanghai, China.

\section{Statistical analysis of diversity}

16S rRNA sequence quality filtering was conducted using Cutadapt (V. 1.9.1; https://cutadapt.readthedocs.io/en/stable/) (Martin, 2011). Quality filtered paired-end reads were then merged using UCHIME (http://www.drive5.com/usearch/manual/uchime_algo.html) [24]. The highquality sequences were clustered into operational taxonomic units (OTUs) at the $97 \%$ nucleotide similarity level using UPARSE [24]. Chimeras were identified and removed from the dataset. DADA2 [25] was used to BLAST representatives of OTUs against the Silva database (https://www.arb-silva.de/) to obtain taxonomic information of each OTU [26]. OTU subsampling was conducted to facilitate comparison amongst samples. Spearman correlation analysis was conducted for OTU abundances across samples, and only robust (Spearman's $r>0.6$ or $r<-0.6)$ and statistically significant $(p<0.01)$ correlations were retained for network analysis. Network analysis of OTU abundances was conducted using the Psych software for R [27], followed by visualization with GEPHI [28]. Alpha diversity indices (Shannon diversity, Simpson diversity, ACE richness, Chao1 richness, and whole-tree PD) were calculated using QIIME2 [25]. Beta diversity values (Bray-Curtis distances) were analyzed using a principal coordinates analysis (PCoA) [26]. Statistical significance of bacterial community variation among the three regions was evaluated with an analysis of similarities (ANOSIM) test. To evaluate the most discriminatory taxa across samples, the relative abundances of bacterial taxa at the genus level were assessed using the random forest package (V. 4.6 - 14) for $R$ with default parameters [29]. Pairwise comparisons of environmental factors were conducted based on Spearman correlation coefficients that were calculated in $\mathrm{R}[30]$.

\section{Metagenomic analysis}

Metagenomic analysis was used to assess the functional changes of soil microbiomes based on respective forest settings (i.e., ages). PCoA analysis of microbiomes (described above) indicated that samples were segregated based on plantation ages, with three groups being evident (new: one year old trees, middle: three and five years, and old: seven and nine years). Six soil samples from each group were randomly selected for metagenomic sequencing. Whole genome shotgun (WGS) metagenomic 
sequencing was used to sequence total metagenomic DNA on the Illumina Novaseq/Hiseq highthroughput sequencing platforms using 150 bp paired-end sequencing after fragmenting the extracted soil DNA. An average of $12 \mathrm{Gbp}$ per sample was generated from the sequencing libraries. Clean data were obtained by quality filtering the original data using Cutadapt (V. 1.17). Taxonomic annotation of sequence data was conducted using Kraken2, while Megahit was used for assembly [31]. After assembly, contigs of length $<200 \mathrm{bp}$ were removed [32]. Species annotation of assembled contig sequences was integrated with the abundance tables in each sample to obtain species abundance tables at each taxonomic rank (i.e., domain, phylum, class, order, family, genus, and species). The MetaGeneMark software program (http://exon.gatech.edu/GeneMark/) [33] was used to identify prokaryotic open reading frame (ORF) and coding regions in addition to protein annotations. Non-redundant protein sequence sets were compared against the Kyoto Encyclopedia of Genes and Genomes (KEGG) database to annotate gene functions [34].

To better understand the role of planting years in the degradation of soil microbiome functioning, genome-resolved binning analysis was conducted to assess functions among individual populations. Spearman correlations were calculated among KEGG pathway abundances and only robust (Spearman's $r>0.6$ or $r<-0.6)$ and statistically significant $(p<0.01)$ correlations were retained for network analysis. Network analysis of KEGG pathway abundances were performed using the Psych software for R [27] followed by visualization with GEPHI [28] and Sankey diagrams [35].

\section{Soil function validation}

We specifically focused on the KEGG function Ko00943 that was significantly enriched in old C. oleifera plantation soils, relating to plant PSMs degradation. To assess in situ functioning, we treated soils of old C. oleifera plantations with tea saponin $\left(5 \mathrm{~g} \cdot \mathrm{L}^{-1}\right)$. After incubation for 24,48 , and $72 \mathrm{~h}$, the remaining tea saponin contents were analyzed with high performance liquid chromatography (HPLC). To assess more explicitly the in situ functioning of Ko00365, which corresponds to a significantly suppressed carbon cycling pathway in soil microbiomes of Camellia plantations, we mixed furfural $\left(5 \mathrm{~g} \cdot \mathrm{L}^{-1}\right)$, an intermediate product of cellulose degradation in the pathway, in soils of old and new Camellia plantations. After incubation for 24,48 , and $72 \mathrm{~h}$, residual furfural was measured using HPLC. To verify that the core bacterial populations were associated with tea saponin degradation, Acinetobacter were enriched using tea saponin as the single carbon source in media. Tea saponin $\left(5 \mathrm{~g} \cdot \mathrm{L}^{-1}\right)$ as a substrate was then mixed with sterilized soil, after supplementing Acinetobacter cultures. After incubation for 24,48 , and $72 \mathrm{~h}$, residual tea saponin contents in the soils were measured with HPLC. Structural equation modelling (SEM) was used to gain a mechanistic understanding of the direct and indirect factors driving SOM across planting years. SEM analyses of SOM, Planting Years, TS, Acinetobacter, Ko00843 and Ko00365 were established for the main factors related to SOM accumulation. We selected the best model based on overall goodness of fit, including the chi-square $\left(\chi^{2}\right)$ statistic, degrees of freedom (df), whole-model $p$ value, goodness of fit index (GFI), and normed fit index (NFI). SEM analyses were conducted using Amos v.21.0 (IBM). 


\section{Results}

\section{Soil physicochemical properties in different-age C. oleifera plantations}

Soil physicochemical properties clearly differed among $C$. oleifera soils in plantations of various ages. Specifically, SOC, SD, and TS were significantly and positively correlated with Camellia planting years $\left(\mathrm{R}^{2}\right.$ $>0.8, p<0.05$ ) (Fig. S1a, i, k). TN, TP, and pH were also significantly and negatively correlated with increased planting years $\left(\mathrm{R}^{2}>0.75, p<0.05\right)$ (Fig. S1b, $\mathrm{C}, \mathrm{h}$ ). A positive correlation was observed between SW and planting years $\left(\mathrm{R}^{2}=0.59, p<0.05\right)$ (Fig. S1j). AP, TK, and AK correlated moderately. However, AP and TK initially increased with planting years and then decreased, while AK content fluctuated among groups (Fig. S1e, $f, g$ ). The accumulation of tea saponin with increased planting years was significantly and positively correlated with SD and SW (Fig. 1c). In contrast, TN, TP, and pH were negatively correlated with tea saponin accumulation.

\section{Effects of C. oleifera plantation ages on soil microbiome structures and diversities}

Co-occurrence network analysis indicated that the species composition of the soil microbiomes significantly changed with increasing planting years. Obviously dominant flora was not present in the soil microbiomes of 1- to 5-year-old plantations, and significant differences were not observed in the proportions of each genus that represented between $2 \%$ and $7 \%$ of the overall communities (Fig. 1a). They began to appear in the 7- to 9-year-old C. oleifera plantation soils. Specifically, Enterobacter (50.41\%), Acinetobacter (11.79\%), and Serratia (4.07\%) became dominant in soils after the seventh year of $C$. oleifera cultivation. Acinetobacter abundance further increased in nine-year-old plantations (14.36\%) (Fig. 1a). Bacterial richness and network complexity gradually decreased from the first year (with an average degree of 40.51) to the fifth year (with an average degree of 34.96) and then to the ninth year (with an average degree of 25.49), with the latter representing the lowest richness and network complexity (Fig. 1a, Table 1). The number of 'hub nodes' (nodes with high degree values, > 60; and closeness centrality, >0.3) in the network gradually decreased with increasing planting years (Fig. 1a; Table 1). 
Table 1

Bacterial co-occurrence network characteristics of $C$. oleifera planting years

\begin{tabular}{|llllllll|}
\hline Niche/Year & Node & $\begin{array}{l}\text { Positive } \\
\text { edge }\end{array}$ & $\begin{array}{l}\text { Negative } \\
\text { edge }\end{array}$ & $\begin{array}{l}\text { Average } \\
\text { degree }\end{array}$ & Modularitya & $\begin{array}{l}\text { Average } \\
\text { clustering } \\
\text { coefficient }\end{array}$ & $\begin{array}{l}\text { Average } \\
\text { path } \\
\text { distance }\end{array}$ \\
\hline 1 & 524 & 7234 & 3379 & 40.508 & 1.169 & 0.591 & 2.951 \\
\hline 3 & 524 & 6773 & 2660 & 35.966 & 1.173 & 0.54 & 2.779 \\
\hline 5 & 462 & 4501 & 1523 & 34.694 & 1.0968 & 0.625 & 2.513 \\
\hline 7 & 246 & 3005 & 1152 & 33.797 & 1.062 & 0.664 & 2.429 \\
\hline 9 & 195 & 1720 & 765 & 25.487 & 1.319 & 0.682 & 2.321 \\
\hline
\end{tabular}

Thus, $C$. oleifera tree age had a strong effect on bacterial alpha diversity (i.e., based on Shannon, Simpson, Chao1, and observed richness indices) in addition to network complexity (i.e., a higher average degree representing a greater network complexity) (Fig. b and c). Among soil physicochemical properties, SOM, pH, SD, SW, and TS were negatively correlated with the four alpha diversity indices (Fig. 1C). PCoA analysis of soil microbiomes were conducted using Bray-Curtis distances and indicated that soil microbiome samples from across planting years could be clustered into three groups. The first group included soil samples after the first year of planting, while the second group included samples of 3 - 5year-old, and the third group included samples from 7 - to 9-year-old plantations (Fig. 1d and Table 2). Random forest classification was used to identify discriminatory taxa for soils of different-age plantations, revealing that Enterobacter, Acinetobacter, Aquabacterium, and Rhodoplanes were significantly enriched in 7 - 9-year plantation soil microbiomes (Fig. 1e). 
Table 2

Intra-group and inter-group Adonis analysis based on Bray_curtis distance

\begin{tabular}{|c|c|c|c|c|c|c|c|}
\hline Treatments & Groups & Df & SumsOfSqs & MeanSqs & F.Model & $\mathrm{R}^{2}$ & $P$ value \\
\hline \multirow[t]{3}{*}{1 Year } & Intra-group & 2 & 1.86 & 0.93 & 7.17 & 0.49 & \multirow[t]{3}{*}{0.001} \\
\hline & Inter-group & 15 & 1.95 & 0.13 & & 0.51 & \\
\hline & Total & 17 & 3.81 & & & 1.00 & \\
\hline \multirow[t]{3}{*}{3 Year } & Intra-group & 2 & 1.66 & 0.83 & 5.53 & 0.42 & \multirow[t]{3}{*}{0.001} \\
\hline & Inter-group & 15 & 2.25 & 0.15 & & 0.58 & \\
\hline & Total & 17 & 3.92 & & & 1.00 & \\
\hline \multirow[t]{3}{*}{5 Year } & Intra-group & 2 & 3.11 & 1.56 & 12.32 & 0.62 & \multirow[t]{3}{*}{0.001} \\
\hline & Inter-group & 15 & 1.90 & 0.13 & & 0.38 & \\
\hline & Total & 17 & 5.01 & & & 1.00 & \\
\hline \multirow[t]{3}{*}{7 Year } & Intra-group & 2 & 0.18 & 0.09 & 5.00 & 0.60 & \multirow[t]{3}{*}{0.001} \\
\hline & Inter-group & 15 & 0.27 & 0.02 & & 0.40 & \\
\hline & Total & 17 & 0.45 & & & 1.00 & \\
\hline \multirow[t]{3}{*}{9 Year } & Intra-group & 2 & 0.11 & 0.06 & 9.63 & 0.56 & \multirow[t]{3}{*}{0.001} \\
\hline & Inter-group & 15 & 0.09 & 0.01 & & 0.44 & \\
\hline & Total & 17 & 0.20 & & & 1.00 & \\
\hline
\end{tabular}

Correlation analysis of the relative abundances of these four genera and soil physicochemical indices demonstrated that the accumulation of tea saponin was significantly and positively correlated with increased Acinetobacter relative abundances (Fig. 1f). Age increase of $C$. oleifera plantation was associated with more similar soil microbiome compositions among different regions (Fig. 2 and Table 2). As indicated above, soil microbiome samples could be clustered into three groups according to bacterial community similarity (Fig. 2 and Table 2). Further, soil microbiome community composition became homogenous over time, with soils from 7- to 9-year-old $C$. oleifera plantations exhibiting the highest intragroup similarities (Fig. 2 and Table 2).

\section{Effects of C. oleifera plantation ages on soil microbiome functions}

Functional analysis of soil microbiomes was conducted by analyzing new ( 1 year), middle ( 3 and 5 year), and old (7 and 9 year) plantation age soil microbiomes. Co-occurrence network analysis based on KEGG annotations indicated that new $C$. oleifera plantations exhibited the highest average degree of soil functional composition (85.519). The density of functional components in soil communities significantly 
decreased with time (with an average degree of 19.178 in new plantations), wherein the functional composition of old C. oleifera plantations was least dense (with average degree of 18.592) (Fig. 3 and Table 3). In the soil functional network for new Camellia plantations, four modules comprised greater than $10 \%$ of the overall network including modules 7 (30.19\%), 11 (23.05\%), 1 (21.75\%), and 0 (15.58\%). Among the three groups, node clustering degree was highest in the new plantations (with average clustering coefficient of 0.669). The compositions of modules changed in the middle-age plantations, wherein modules 2 (33.19\%), 6 (28.63\%), 1 (21.75\%), and 0 (15.58\%) accounted for over $10 \%$ of the entire network. In particular, the first two modules were different than those in the new plantation soil networks (Fig. 3 and Table 3). Only the modules with the highest proportion changed compared to the intermediate year networks (module 11, 32.5\%) (Fig. 3 and Table 3).

Table 3

KEGG pathways co-occurrence network characteristics of $C$. oleifera planting years

\begin{tabular}{|c|c|c|c|c|c|c|c|}
\hline Niche/Year & Node & $\begin{array}{l}\text { Positive } \\
\text { edge }\end{array}$ & $\begin{array}{l}\text { Negative } \\
\text { edge }\end{array}$ & $\begin{array}{l}\text { Average } \\
\text { degree }\end{array}$ & Modularity ${ }^{a}$ & $\begin{array}{l}\text { Average } \\
\text { clustering } \\
\text { coefficient }^{b}\end{array}$ & $\begin{array}{l}\text { Average } \\
\text { path } \\
\text { distance }\end{array}$ \\
\hline New & 308 & 8688 & 4482 & 85.519 & 0.964 & 0.669 & 1.872 \\
\hline Middle & 241 & 1429 & 882 & 19.178 & 2.614 & 0.609 & 2.85 \\
\hline Old & 240 & 1627 & 604 & 18.592 & 1.049 & 0.603 & 2.849 \\
\hline
\end{tabular}

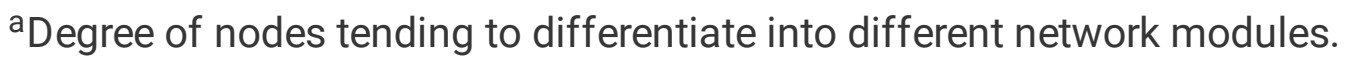

${ }^{b}$ Degree of nodes tending to cluster together.

cNetwork path distance is the length of the shortest path between two nodes within the network.

Analysis of KEGG level 1 annotations indicated that metabolism was the core functional category represented among the microbiomes of all three plantation age groups, while comparison of KEGG level 2 annotations revealed differences in core functions among soils of different aged plantations. The functions within new plantation soil microbiomes primarily comprised the categories of signal transduction (7.14\%), metabolism of terpenoids and polyketides (6.82\%), xenobiotics biodegradation and metabolism (6.49\%), biosynthesis of other PSMs (6.17\%), lipid metabolism (5.19\%), carbohydrate metabolism (4.87\%), glycan biosynthesis and metabolism (4.87\%), and amino acid metabolism (4.55\%) (Fig. 3). In middle aged plantation soils, xenobiotics biodegradation and metabolism (8.3\%) in addition to metabolism of terpenoids and polyketides $(7.47 \%)$ became more prominent (Fig. 3). Lastly, xenobiotics biodegradation and metabolism (8.33\%) in addition to terpenoids and polyketides $(7.08 \%)$ were similarly higher in old $C$. oleifera plantation soils. In addition, the biosynthesis of other secondary metabolites (6.25\%) and carbohydrate metabolism (6.25\%) gradually replaced the core functions of new Camellia 
plantation microbiomes and became core functional pathways in the soil microbiomes of old ones (Fig. $3)$.

To better understand the effects of plantation years on differences in soil microbiome functions, KEGG pathway abundances were compared among the three different-age plantation soils. Differences were not observed among groups in the proportions of the ten most abundant KEGG pathway groups. Overall, seventy-one pathways were significantly enriched in soil samples from new Camellia plantations, while one pathway was enriched in old plantation soils, and no pathways were significantly enriched in middleage plantation soils (Fig. 4b and c). We further analyzed KEGG pathway differences between old and new Camellia plantation soils, observing the significant enrichment of the Ko00984 pathway (KEGG level 3: steroid degradation) in old plantation soils, while the other 19 pathways were enriched in new plantation soils, as described above (Fig. 4c). Ko00365 (KEGG level 3: furfural degradation) is an important pathway involved in cellulose degradation and was significantly enriched in new plantation soils, but in low abundance in old plantation soils (Fig. 4e). Ko00984 is an important pathway involved in PSM degradation via the continuous decomposition of tea saponin into smaller molecular weight compounds (Fig. 4d). Genomic binning analysis was subsequently used to assemble genomes from the metagenomes of old and new C. oleifera plantation soils to better assess the context of the Ko00984 and Ko00365 pathways. Sankey diagram visualization indicated that the Ko00984 pathway could be assigned to nine bacterial genomes, with two belonging to Bacillus and the other seven Acinetobacter. In addition, the Ko00365 pathway was attributed to four bacterial genomes belonging to Burkholderia, Paraburkholderia, Pseudomonas, and Kelebsiella (Fig. 4f). SEM model showed that the spatial variation of SOM was influenced by planting years, tea saponin content, Acinetobacter, Ko00842 and Ko00365, and $97.5 \%$ of SOM spatial variation was explained by SEM model. All the indexes can directly affect the SOM: cultivated fixed number of year $(\beta=0.984)$, tea saponin content $(\beta=0.196)$, Acinetobacter $(\beta=$ 0.309 ) and Ko00842 (can directly increase SOM $(\beta=0.081)$, While Ko00365 $(\beta=-0.062)$ can directly reduce SOM. Planting years, tea saponin content and Acinetobacter also have indirect effects on SOM. It is worth noting that planting years can indirectly affect SOM by affecting tea saponin content and Acinetobacter and can also indirectly affect SOM by affecting tea saponin content, Ko00842 or Ko00365 (Fig. 6).

\section{Functional verification of Acinetobacter activities}

Degradation experiments demonstrated that new $C$. oleifera plantation soils exhibited higher furfural degradation efficiencies than the old ones (Fig. 5a). Further, furfural degradation was significantly different after $12 \mathrm{~h}$ of degradation experiments ( $t$-test, $\mathrm{t}=3.02, \mathrm{df}=8, p<0.05$ ) (Fig. $5 \mathrm{~b}$ ). The efficiency of tea saponin degradation was however higher in old $C$. oleifera forest soils (Fig. $5 \mathrm{C}$ ), although degradation was relatively slow. Specifically, differences between treatment and control incubations were only significant after $48 \mathrm{~h}$ ( $t$-test, $\mathrm{T}=4.85, \mathrm{df}=8, p<0.05$ ) (Fig. $5 \mathrm{~d}$ ). Moreover, isolated Acinetobacter exhibited strong tea saponin degradation abilities (Fig. $5 \mathrm{~d}$ ). After $12 \mathrm{~h}$ of culture, the efficiency of tea saponin degradation in soils with Acinetobacter were significantly different from those of $\mathrm{CK}(\mathrm{t}$-test, $\mathrm{T}=7.90, \mathrm{df}=$ $8, p<0.05)$ (Fig. 5e). 


\section{Discussion}

Soil properties gradually deteriorated with age-increasing of C. oleifera plantations

Density and water content are important indices of soil water storage capacity. Lower soil density represents greater soil porosity, resulting in better soil structure and performance along with water storage capacity [4]. Older $C$. oleifera plantations led to increased litter and canopy densities that could improve soil conditions and promote the formation of soft soils (Fig. 1c and Fig. S1). Nevertheless, soil densities of old $C$. oleifera plantations were significantly higher than those of new ones, while the organic matter contents were also higher (Fig. 1c and Fig. S1). This indicated that litter decomposition was suppressed in old plantations, and the release of t organic matter, nitrogen, phosphorus, and other substances into soils, along with PSMs was inhibited [3-4,36]. Consequently, soil contents of total nitrogen and phosphorus continuously drop, while PSM (i.e., tea saponin) accumulation significantly keeps increasing (Fig. 1c and Fig. S1). The lack of artificial supplementation leads to the demand for $\mathrm{N}$ and $\mathrm{P}$ by plants being a primary reason for the significant decrease with the time being (Fig. 1c and Fig. S1). In contrast, the decomposition rate of litters with higher contents of structural carbohydrates like tea saponin was slower, although these PSMs may significantly accumulate within the soil from age-increasing plantations $[3,48]$.

\section{Concentration of tea saponin serving the primary factor affecting soil microbiome structures}

Tea saponin is important PSM of $C$. oleifera and belong to the triterpenoid class of saponins [37]. Its accumulation inhibited soil microbial decomposition on plant litters. The litter contains diverse PSMs including alkaloids, phenolic compounds, and terpenes, all of which are often used an against herbivores and pathogens, thereby playing key roles in plant-microbe and plant-herbivore interactions [3]. Tannins may slow rates of litter decomposition [38], and phenolic compounds can delay the colonization of litter by decomposer organisms $[3,39]$. Problematic root-derived phenolics in soils can also drive shifts in microbial community compositions and prime decomposition activities in forest soils [40]. Age-ncreasing plantation was associated with gradual decreases in soil microbiome diversity and their structures became simpler (Fig. 1a-c). Further, soil microbial communities exhibited similarities with the increase of plantation ages (Fig. 2) and tea saponin contents were the primary factor driving this transition (Fig. 1C). Our results agree with other studies. Schütz et al (2021) [41] disclosed that plant-derived PSMs and their derivatives may contribute to the regulation of plant microbiomes and their functional diversity and a considerable amount of allelopathic and microbiota-modifying metabolites is released from plant litter, thereby influencing soil microbial communities. Further, benzoxazinoids have been long known to exhibit allelopathic properties, including microbial degradation products in soils [42].

Tea saponin is also an important fungicide composed of sugar chains, triterpenoids, steroids, or steroid alkaloids that are linked by carbon-oxygen bonds [21-22]. The structures of insect gut microbiomes have been significantly changed after feeding on fruits containing tea saponin [21]. The soil microbiomes 
evaluated here also exhibited similar changes in response to saponin contents. Moreover, the abundances of Enterobacter, Acinetobacter, Aquabacterium, and Rhodoplanes were associated with the increasing tea saponin accumulation that accrued across plantation ages (Fig. 1e and f). In addition, Acinetobacter abundance was significantly and positively correlated with tea saponin accumulation (Fig. 1f). The bacterium is frequently associated with both aspen foliage and the gypsy moths that consume aspen foliage tissue, metabolizing phenolic glycosides within the foliage [43]. Further, species of $A$. calcoaceticus and A. oleivorans were able to degrade catechin, modulating host physiology and metabolism to improve hexadecane utilization efficiency [44]. Acinetobacter derived from wood-fed termite guts can efficiently degrade phenolic compounds by using phenol as its sole carbon source [45]. Community interaction networks and random forest classification analyses conducted in the study indicated Acinetobacter was one of the core soil bacteria in old $C$. oleifera plantations. The relative abundances of Acinetobacter were significantly and positively correlated with tea saponin accumulation, firmly suggesting that Acinetobacter closely involved in tea saponin degradation.

\section{Accumulation of tea saponin affecting soil decomposition functions}

Most plant species whose litter releases terpenoids, phenolics, steroids, and aliphatic acids generally inhibit the litter decomposition and/or nutrient releasing $[1,46]$. Comparison of soil microbiome functional profiles from $C$. oleifera plantations of different ages at three sites revealed that metabolism was the primary KEGG level 1 function (Fig. 3). Correlation analysis and subsequent network analysis indicated that functional modules became less connected with the increase of plantation ages, while the predominant KEGG level 2 function changed among groups (Fig. 3). In the old plantation age soils, functional modules were primarily annotated as xenobiotics biodegradation and metabolism; terpenoids and polyketides; and biosynthesis of other secondary metabolites (Fig. 3). As discussed above, tea saponin accumulation significantly altered soil microbial populations in the old $C$. oleifera plantation soils, corresponded to changes in community functions. Pathways involved in nitrogen and phosphorus cycling's that are beneficial to soil quality improvement were not significantly enriched. Similarly, the study on impacts of continuous crop planting on soil functions indicated that pathways involved in nitrogen and phosphorus cycling were negatively affected, leading to crop yield reduction [47].

PSMs enter soils and significantly influence soil function, following litter decomposition [48-52]. Our results indicated that few KEGG pathways primarily for steroid degradation were significantly enriched in the soil of old $C$. oleifera plantations with high tea saponin content (Fig. 4c and d). During decomposition process, tea saponin is hydrolyzed into numerous steroids, glycosides, and other substances, and the enrichment of the Ko00984 pathway could be related to the degradation of tea saponin. Soil microorganisms may participate in the decomposition of tea saponin via enzymatic activities After tea saponin-derived PSMs enter soils, (Fig. 4c and d). To verify this hypothesis, we used soils from old and new? C. oleifera plantations to conduct tea saponin fermentation degradation experiments, observing a much higher degradation efficiency in old compared to new plantations (Fig. $5 c$ and d). 
In addition, numerous pathways related to nutrient anabolism were significantly less abundant in soil of old C. oleifera plantations, and this was especially evident for the Ko00365: furfural degradation pathway that is closely involved in cellulose degradation (Fig. 4c and e). The litters contain abundant cellulose initially degraded into hemicellulose $s$ then furfural, eventually becoming an important component of SOM. Cellulose passes through soil bacteria via soil respiration, eventually being decomposed into carbon dioxide and water to complete carbon mineralization. In old $C$. oleifera plantation soils, the decomposition of furfural into organic acids was inhibited (Fig. 4c and e) probably due to the accumulation of SOM (Fig. S1a). Soils from old and new C. oleifera plantations were used in furfural decomposition experiments to assess. Results disclosed that the decomposition efficiencies of furfural in soils of new C. oleifera plantations were higher than (Fig. 5a and b), indicating that the accumulation of tea saponin and other PSMs in old $C$. oleifera plantations inhibited increasing of soil bacterial populations involved in cellulose degradation. This apparent inhibition of cellulose degradation pathways could be one of the reasons underlying the continuous accumulation of SOM with plantation age.

In addition, the Ko00984 pathway was primarily enriched in the genomes of Acinetobacter bacteria, while the Ko00365 pathway was primarily enriched in the genomes of bacteria involved in nutrient degradation (Fig. 4F). To confirm whether Acinetobacter can degrade tea saponin, an Acinetobacter strain was cultured from medium containing tea saponin as sole carbon source and their ability to degrade tea saponin was confirmed (Fig. 5E and F). The structural equation model also showed that planting years directly affected tea saponin accumulation, Acinetobacter abundance, and SOM, while tea saponin affected SOM through Acinetobacter abundance and Ko00843 pathway (Fig. 5E and F; Fig. 6). Thus, the enrichment of Acinetobacter in old $C$. oleifera plantations soil considered closely related to degradation of PSMs such as tea saponin (Fig. 5E and F; Fig. 6).

\section{Conclusions}

In this study, the effects of $C$. oleifera plantation ages on soil properties, microbiome structures and functions were explored. Increase of plantation age led to significantly decreased quality in soil nutrient indices, while SOM and soil density increased dramatically. This could be the result of increased accumulation of tea saponin, an important PSM of $C$. oleifera. Tea saponin exhibits bactericidal toxicity, and its accumulation would be lethal to some soil bacteria involved in litter degradation. These dynamics would then affect pathways involved with SOM degradation, resulting in increasing of SOM. The homogenization of soil microbiomes weakens soil decomposition function, increases soil density, and causes soil compaction. Concomitantly, plant growth requires abundant nitrogen and phosphorus nutrients. Thus, when total nitrogen and phosphorus contents in soil decrease over time, old $C$. oleifera plantation soil becomes barren, and productivity drops remarkably. Correct measures should therefore be taken for sustainable management of old $C$. oleifera plantations. First, functional bacterial fertilizers that could change the soil microbial community structures should be applied in old $C$. oleifera plantations to accelerate litter decomposition. Second, the total nitrogen and phosphorus contents should be increased following the formula to enhance the productivity. Further, these measures are taken together with 
planting of selected understory plants in old Camellia plantations to achieve the goal of sustainable management.

\section{Declarations}

\section{Acknowledgements}

We would like to thank Prof. Jinping Shu for their cooperation and assistance in the field work, and $\mathrm{Yi}$ Wang, Linxin Fang, Hao Yin and Zikun Li for their generous help in soil sampling and laboratory work. We also thank Yongchun Li and Junhao Huang for their suggestions and comments on the manuscript.

\section{Founding}

This work was financially supported by the Launching Funds for Talents of Zhejiang A \& F University (2020FR036), and the Launching Funds for Talents of State Key Laboratory of Subtropical Silviculture, Zhejiang A \& F University (ZY20210201), China.

\section{Availability of data and materials}

Demultiplexed sequence data are available in the NCBI Sequence Read Archive (Bio-Project ID of soil microbiomes: PRJNA772371; Bio-Project ID of soil metagenome: PRJNA772552).

\section{Ethics approval and consent to participate}

Not applicable.

\section{Consent for publication}

Not applicable.

\section{Competing interests}

The authors declare that they have no competing interests.

\section{Author details}

Z-SK, K-JQ, G-K and Z-XD designed the study. Z-SK and H-XH managed the field trial stations. Z-SK and H$\mathrm{XH}$ collected samples. Z-SK and K-JQ conducted the laboratory analyses. Z-SK, K-JQ and C-LF performed the data processes. Z-SK and K-JQ wrote the manuscript. All authors read and approved the final manuscript.

\section{References}

1. Jacoby RP, Koprivova A, Kopriva S. Pinpointing secondary metabolites that shape the composition and function of the plant microbiome. Journal of Experimental Botany. 2021;72(1):57-69. 
2. Yang X, Wang X, Xiao S, Liu Z, Zhou X, Du G, et al. Dominant plants affect litter decomposition mainly through modifications of the soil microbial community. Soil Biology and Biochemistry. 2021:108399.

3. Chomel M, Guittonny-Larchevêque M, Fernandez C, Gallet C, DesRochers A, Paré D, et al. Plant secondary metabolites: a key driver of litter decomposition and soil nutrient cycling. Journal of Ecology. 2016;104(6):1527-41.

4. Veen G, Fry EL, ten Hooven FC, Kardol P, Morriën E, De Long JR. The role of plant litter in driving plantsoil feedbacks. Frontiers in Environmental Science. 2019;7:168.

5. Dror B, Jurkevitch E, Cytryn E. State-of-the-art methodologies to identify antimicrobial secondary metabolites in soil bacterial communities-A review. Soil Biology and Biochemistry. 2020;147:107838.

6. Rogers EE, Glazebrook J, Ausubel FM. Mode of action of the Arabidopsis thaliana phytoalexin camalexin and its role in Arabidopsis-pathogen interactions. Molecular Plant Microbe Interactions. 1996;9:748-57.

7. De León L, López M, Moujir L. Antibacterial properties of zeylasterone, a triterpenoid isolated from Maytenus blepharodes, against Staphylococcus aureus. Microbiological Research. 2010;165(8):617-26.

8. Borges A, Abreu AC, Ferreira C, Saavedra MJ, Simões LC, Simões M. Antibacterial activity and mode of action of selected glucosinolate hydrolysis products against bacterial pathogens. Journal of food science and technology. 2015;52(8):4737-48.

9. Yang L, Ding W, Xu Y, Wu D, Li S, Chen J, et al. New insights into the antibacterial activity of hydroxycoumarins against Ralstonia solanacearum. Molecules. 2016;21(4):468.

10. Hu L, Robert CA, Cadot S, Zhang X, Ye M, Li B, et al. Root exudate metabolites drive plant-soil feedbacks on growth and defense by shaping the rhizosphere microbiota. Nature communications. 2018;9(1):1-13.

11. Stringlis IA, Yu K, Feussner K, De Jonge R, Van Bentum S, Van Verk MC, et al. MYB72-dependent coumarin exudation shapes root microbiome assembly to promote plant health. Proceedings of the National Academy of Sciences. 2018;115(22):E5213-E22.

12. Cotton TA, Pétriacq P, Cameron DD, Al Meselmani M, Schwarzenbacher R, Rolfe SA, et al. Metabolic regulation of the maize rhizobiome by benzoxazinoids. The ISME journal. 2019;13(7):1647-58.

13. Huang AC, Jiang T, Liu Y-X, Bai Y-C, Reed J, Qu B, et al. A specialized metabolic network selectively modulates Arabidopsis root microbiota. Science. 2019;364(6440).

14. Kudjordjie EN, Sapkota R, Steffensen SK, Fomsgaard IS, Nicolaisen M. Maize synthesized benzoxazinoids affect the host associated microbiome. Microbiome. 2019;7(1):1-17.

15. Kamau S, Barrios E, Karanja NK, Ayuke FO, Lehmann J. Dominant tree species and earthworms affect soil aggregation and carbon content along a soil degradation gradient in an agricultural landscape. Geoderma. 2020;359:113983.

16. Wang S, Zuo X, Zhao X, Awada T, Luo Y, Li Y, et al. Dominant plant species shape soil bacterial community in semiarid sandy land of northern China. Ecology and evolution. 2018;8(3):1693-704. 
17. Wu F, Li J, Chen Y, Zhang L, Zhang Y, Wang S, et al. Effects of phosphate solubilizing bacteria on the growth, photosynthesis, and nutrient uptake of Camellia oleifera Abel. Forests. 2019;10(4):348.

18. Zhang T, Qiu F, Chen L, Liu R, Chang M, Wang X. Identification and in vitro anti-inflammatory activity of different forms of phenolic compounds in Camellia oleifera oil. Food Chemistry. 2021;344:128660.

19. Liu J, Wu L, Chen D, Li M, Wei C. Soil quality assessment of different Camellia oleifera stands in midsubtropical China. Applied Soil Ecology. 2017;113:29-35.

20. Yuan J, Huang L, Zhou N, Wang H, Niu G. Fractionation of inorganic phosphorus and aluminum in red acidic soil and the growth of Camellia oleifera. HortScience. 2017;52(9):1293-7.

21. Zhang S, Shu J, Xue H, Zhang W, Zhang Y, Liu Y, et al. The Gut Microbiota in Camellia Weevils Are Influenced by Plant Secondary Metabolites and Contribute to Saponin Degradation. Msystems. 2020;5(2):e00692-19.

22. Cui C, Yang Y, Zhao T, Zou K, Peng C, Cai H, et al. Insecticidal activity and insecticidal mechanism of total saponins from Camellia oleifera. Molecules. 2019;24(24):4518.

23. Jiang S, Xing Y, Liu G, Hu C, Wang X, Yan G, et al. Changes in soil bacterial and fungal community composition and functional groups during the succession of boreal forests. Soil Biology and Biochemistry. 2021:108393.

24. Edgar, R. C., Haas, B. J., Clemente, J. C., Quince, C., \& Knight, R. UCHIME improves sensitivity and speed of chimera detection. Bioinformatics. 2011; 27(16): 2194-2200.

25. Bolyen E, Rideout JR, Dillon MR, Bokulich NA, Abnet CC, Al-Ghalith GA, et al. Reproducible, interactive, scalable and extensible microbiome data science using QIIME 2. Nature biotechnology. 2019;37(8):852-7.

26. Wang Q, Garrity GM, Tiedje JM, Cole JR. Naive Bayesian classifier for rapid assignment of rRNA sequences into the new bacterial taxonomy. Applied and environmental microbiology. 2007;73(16):5261-7.

27. Faust K, Sathirapongsasuti JF, Izard J, Segata N, Gevers D, Raes J, et al. Microbial co-occurrence relationships in the human microbiome. PLoS computational biology. 2012;8(7):e1002606.

28. Bastian M, Heymann S, Jacomy M: Gephi: an open-source software for exploring and manipulating networks. In: Third international AAAI conference on weblogs and social media. 2009.

29. Liaw A, Wiener M. Classification, and regression by randomForest. R news. 2002;2(3):18-22.

30. Sunagawa S, Coelho LP, Chaffron S, Kultima JR, Labadie K, Salazar G, et al. Structure and function of the global ocean microbiome. Science. 2015;348(6237):1261359.

31. Wood DE, Lu J, Langmead B. Improved metagenomic analysis with Kraken 2. Genome biology. 2019;20(1):1-13.

32. Li D, Liu C-M, Luo R, Sadakane K, Lam T-W. MEGAHIT: an ultra-fast single-node solution for large and complex metagenomics assembly via succinct de Bruijn graph. Bioinformatics. 2015;31(10):1674-6. 
33. Zhu W, Lomsadze A, Borodovsky M. Ab initio gene identification in metagenomic sequences. Nucleic acids research. 2010;38(12):e132-e.

34. Kanehisa M, Araki M, Goto S, Hattori M, Hirakawa M, Itoh M, et al. KEGG for linking genomes to life and the environment. Nucleic acids research. 2007;36(suppl_1):D480-D4.

35. Schmidt M. The Sankey diagram in energy and material flow management: Part II: Methodology and current applications. Journal of industrial ecology. 2008;12(2):173-85.

36. Mazzoleni S, Bonanomi G, Incerti G, Chiusano ML, Termolino P, Mingo A, et al. Inhibitory and toxic effects of extracellular self-DNA in litter: a mechanism for negative plant-soil feedbacks? New Phytologist. 2015;205(3):1195-210.

37. Fan L, He Y, Xu Y, Li P, Zhang J, Zhao J. Triterpenoid saponins in tea (Camellia sinensis) plants: biosynthetic gene expression, content variations, chemical identification and cytotoxicity. International Journal of Food Sciences and Nutrition. 2021;72(3):308-23.

38. Hättenschwiler S, Vitousek PM. The role of polyphenols in terrestrial ecosystem nutrient cycling. Trends in ecology \& evolution. 2000;15(6):238-43.

39. Ormeno E, Baldy V, Ballini C, Larchevêque M, Périssol C, Fernandez C. Effects of environmental factors and leaf chemistry on leaf litter colonization by fungi in a Mediterranean shrubland. Pedobiologia. 2006;50(1):1-10.

40. Zwetsloot MJ, Ucros JM, Wickings K, Wilhelm RC, Sparks J, Buckley DH, et al. Prevalent root-derived phenolics drive shifts in microbial community composition and prime decomposition in forest soil. Soil Biology and Biochemistry. 2020;145:107797.

41. Schütz V, Frindte K, Cui J, Zhang P, Hacquard S, Schulze-Lefert P, et al. Differential impact of plant secondary metabolites on the soil microbiota. Frontiers in microbiology. 2021;12:1267.

42. Schulz H, Dunst G, Glaser B. Positive effects of composted biochar on plant growth and soil fertility. Agronomy for sustainable development. 2013;33(4):817-27.

43. Arunachalam A, Pandey HN. Ecosystem restoration of Jhum fallows in Northeast India: microbial C and $\mathrm{N}$ along altitudinal and successional gradients. Restoration Ecology. 2003;11(2):168-73.

44. Van Dexter S, Boopathy R. Biodegradation of phenol by Acinetobacter tandoii isolated from the gut of the termite. Environmental Science and Pollution Research. 2019;26(33):34067-72.

45. Zhang $X$, Wang B, Liu Z. Impacts of plant secondary metabolites from conifer litter on the decomposition of Populus purdomii litter. Journal of Forestry Research. 2019;30(6):2237-45.

46. Pang Z, Dong F, Liu Q, Lin W, Hu C, Yuan Z. Soil metagenomics reveals effects of continuous sugarcane cropping on the structure and functional pathway of rhizospheric microbial community. Frontiers in Microbiology. 2021;12:369.

47. Jackrel SL, Gilbert JA, Wootton JT. The origin, succession, and predicted metabolism of bacterial communities associated with leaf decomposition. MBio. 2019;10(5):e01703-19.

48. Lozovaya VV, Lygin AV, Zernova OV, Ulanov AV, Li S, Hartman GL, et al. Modification of phenolic metabolism in soybean hairy roots through down regulation of chalcone synthase or isoflavone 
synthase. Planta. 2007;225(3):665-79.

49. Mason CJ, Lowe-Power TM, Rubert-Nason KF, Lindroth RL, Raffa KF. Interactions between bacteria and aspen defense chemicals at the phyllosphere-herbivore interface. Journal of chemical ecology. 2016;42(3):193-201.

50. Ormeno-Orrillo E, Vinuesa P, Zuniga-Davila D, Martínez-Romero E. Molecular diversity of native bradyrhizobia isolated from Lima bean (Phaseolus lunatus L.) in Peru. Systematic and applied microbiology. 2006;29(3):253-62.

51. Zhang P, Cui Z, Guo M, Xi R. Characteristics of the soil microbial community in the forestland of Camellia oleifera. PeerJ. 2020;8:e9117.

\section{Figures}


(a)

- Enterobacter - Acinetobacter • Pseudomonas • Aquabacterium • Rhodoplanes $\bullet$ Meyerozyma $\bullet$ Akkermansia $\bullet$ Serratia Others Positive - Negative $\multimap$ Degree

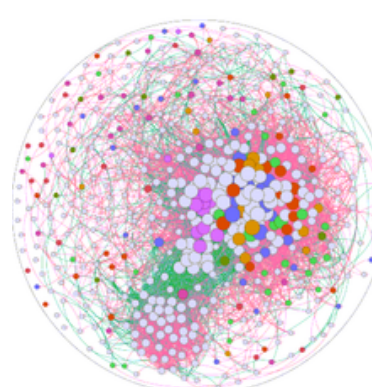

- Module \#5 - Module\#1

Module \#2 Other Modlules

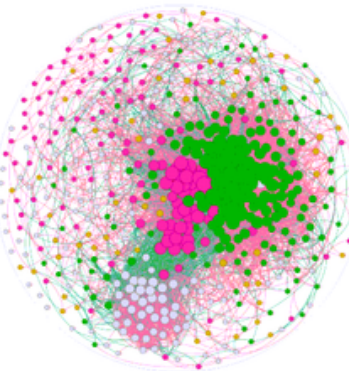

Avg. degree $=40.51$ 1 Year

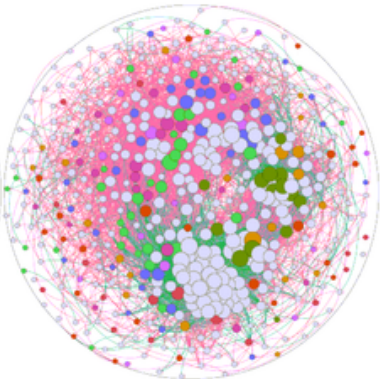

- Module \#4 Module \#2

- Module \#1 Other Modlules

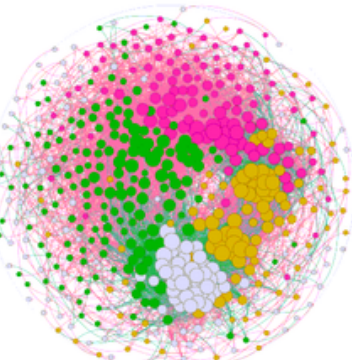

Avg. degree $=35.96$ 3 Year

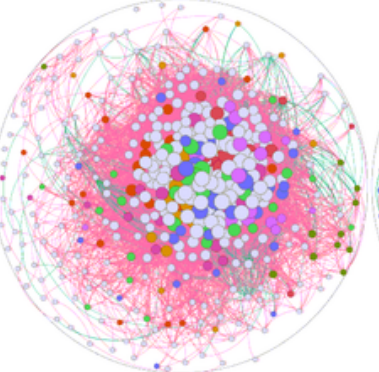

- Module \#0 Module \#2

- Module \#3 Other Modlules

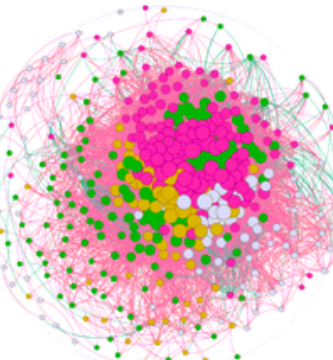

Avg. degree $=34.96$ 5 Year

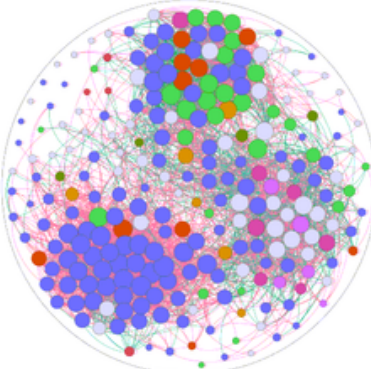

- Module \#1 - Module \#3

- Module \#5 Other Modlules

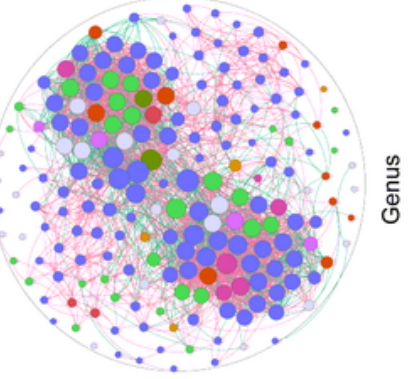

- Module \#4 - Module\#2

- Module \#11 Other Modlules

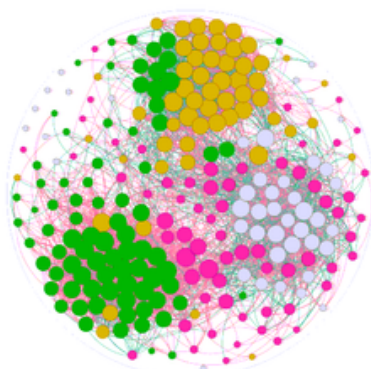

Avg. degree $=33.79$ 7 Year

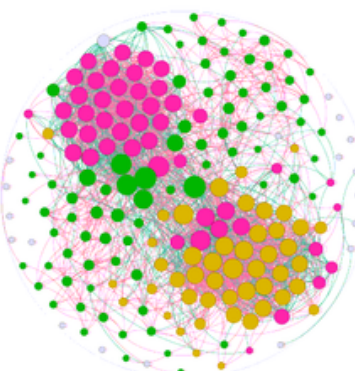

Avg. degree $=25.49$ 9 Year (b)

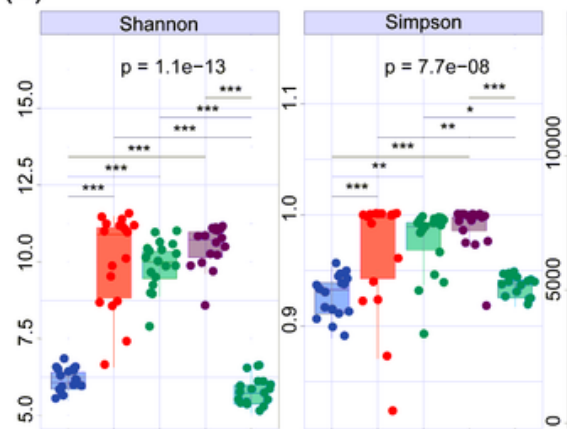

(d)

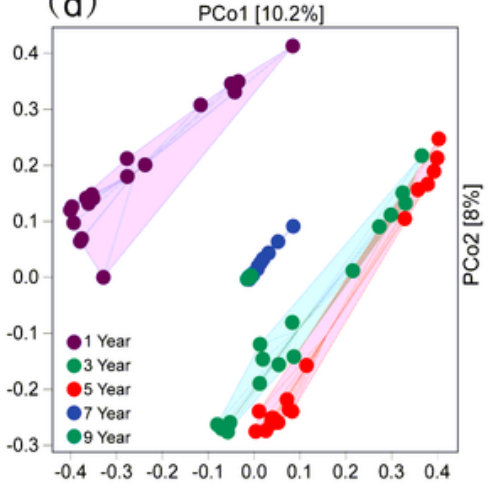

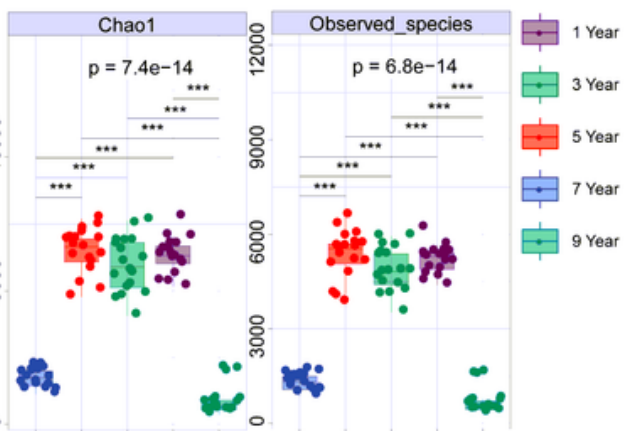

(e)

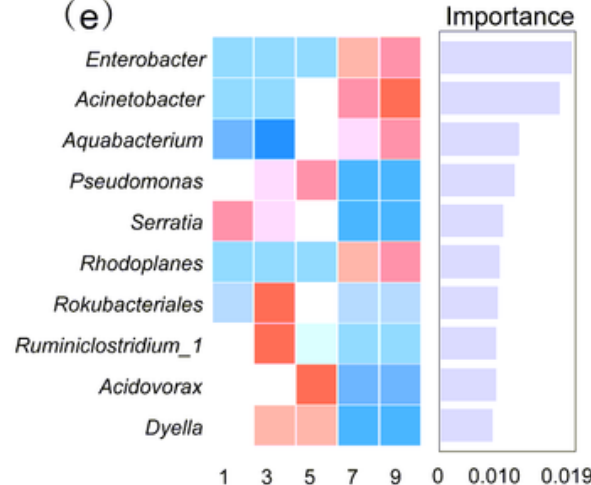

(c)

Planting years

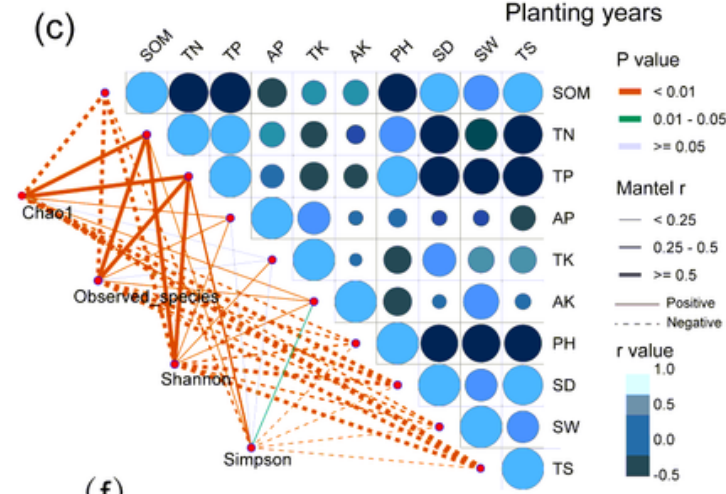

(f)

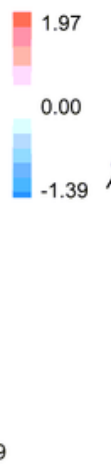

\section{(f)}

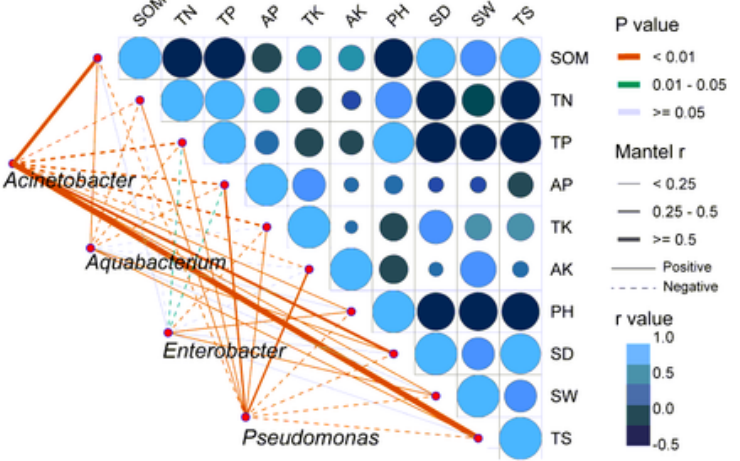

\section{Figure 1}

Effects of C. oleifera plantation ages on soil microbiome structures and diversities. (a). Network of soil microbial populations among $\mathrm{C}$. oleifera plantations ageing from 1 to 9 years. Red lines indicate positive correlations and green lines for negative correlations. The area of the node is proportional to node degree, calculated from correlations of abundances for each ASV. Only correlations with an $r>0.6$ or $<-0.6$ and $p<0.05$ were included in the network. (b). Alpha diversity values for soil microbial communities among 
plantations of various ages. (c). Correlations between soil physicochemical parameters and alpha diversity index values. (d). PCoA analysis of soil community compositional variation based on Bray Curtis distances. (e). Random forest classification analysis of dominant bacteria in plantations of different ages. (f). Correlations between the abundances of dominant bacteria identified by random forest classification as discriminatory for sample groups with soil physicochemical properties.
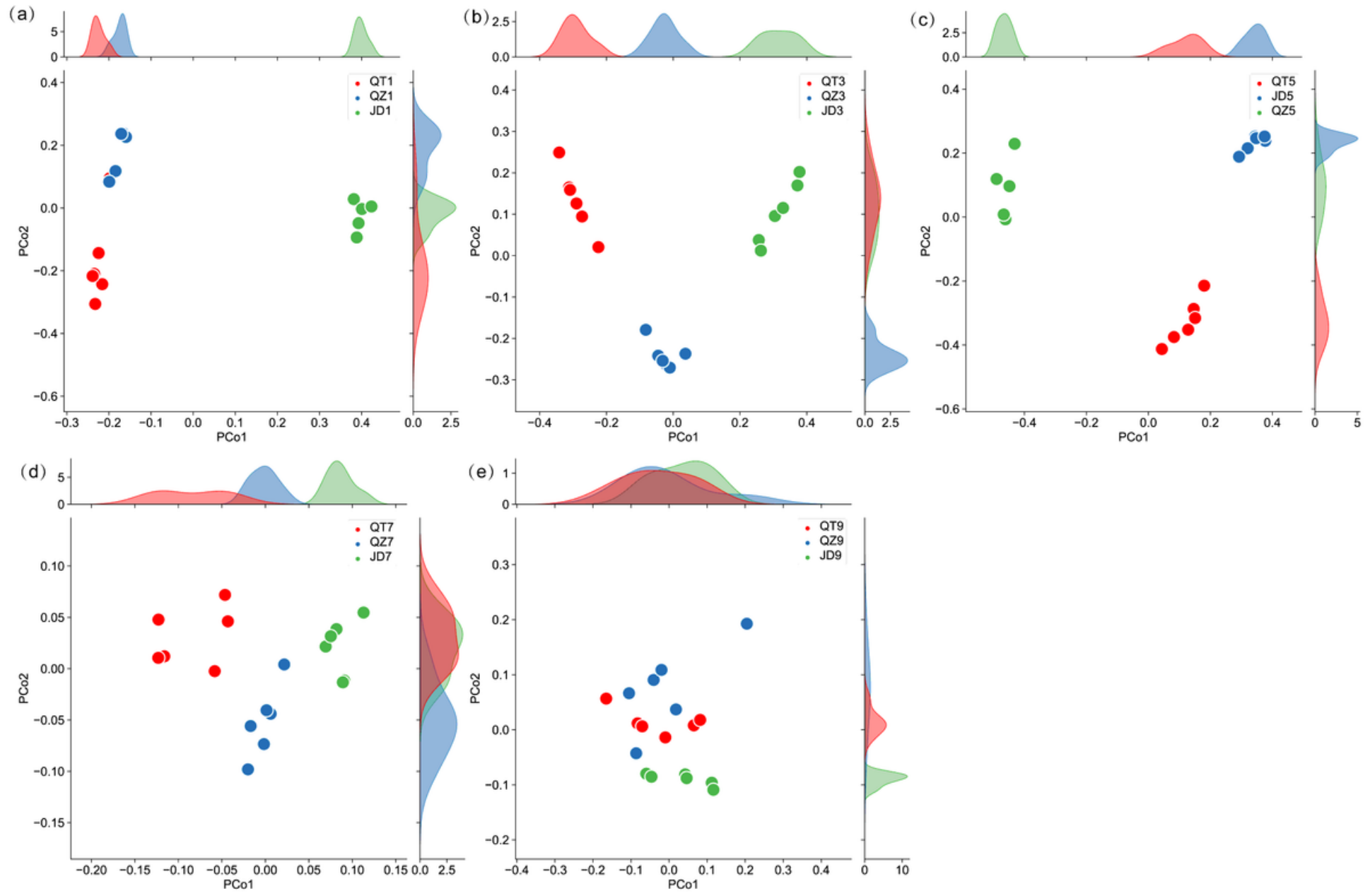

Figure 2

Effects of C. oleifera plantation ages on soil microbial community compositional variation among different sampling sites. 

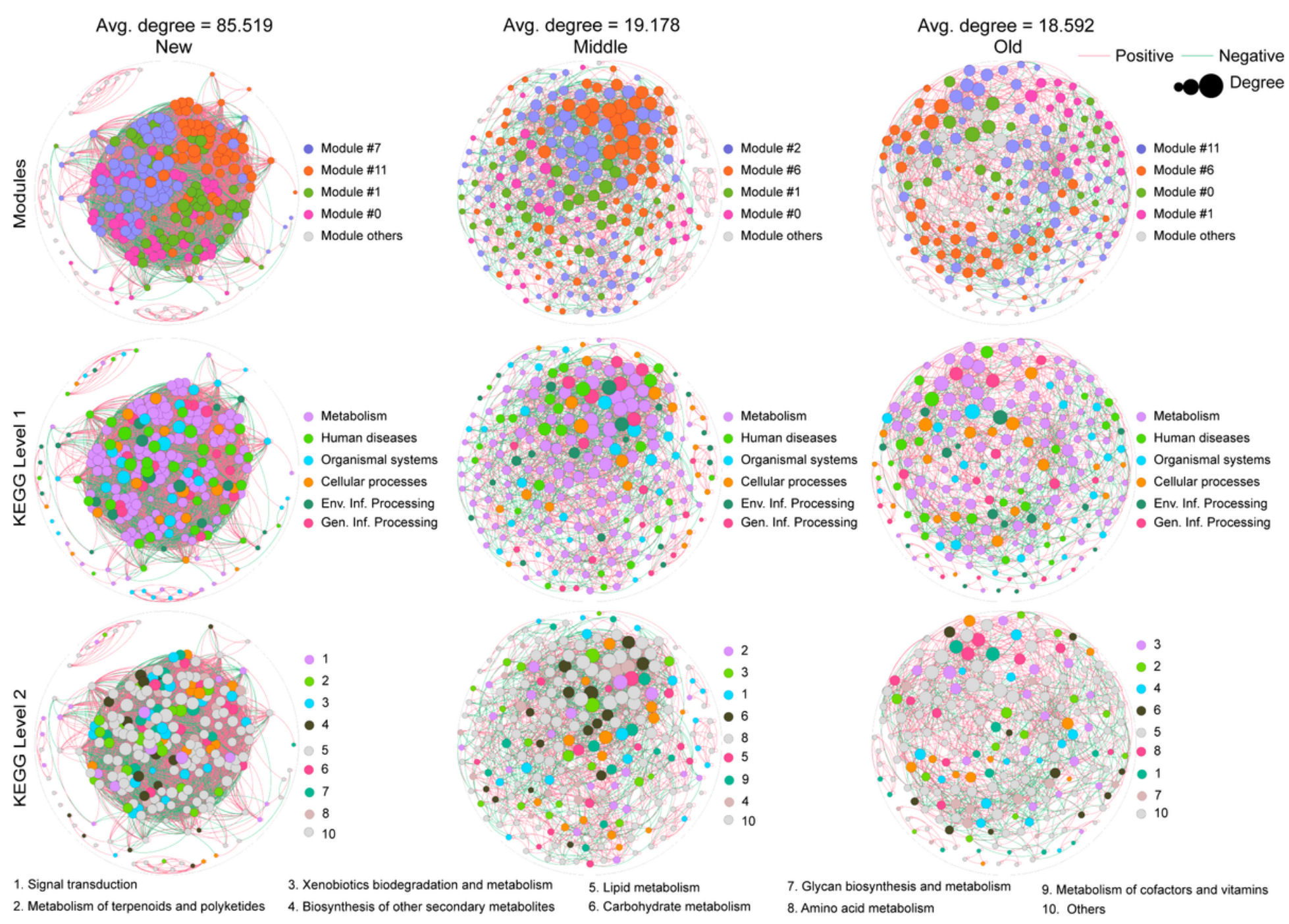

Figure 3

Variation in core soil microbial community functions associated with C. oleifera plantation ages. Functions are based on KEGG annotations. Red lines indicate positive correlations and green lines for negative correlations. The area of the node is proportional to node degree, which is calculated from correlations of abundances for each KEGG pathway. Only correlations with an $r>0.6$ or $<-0.6$ and $p<$ 0.05 were included in the network. 
(a)

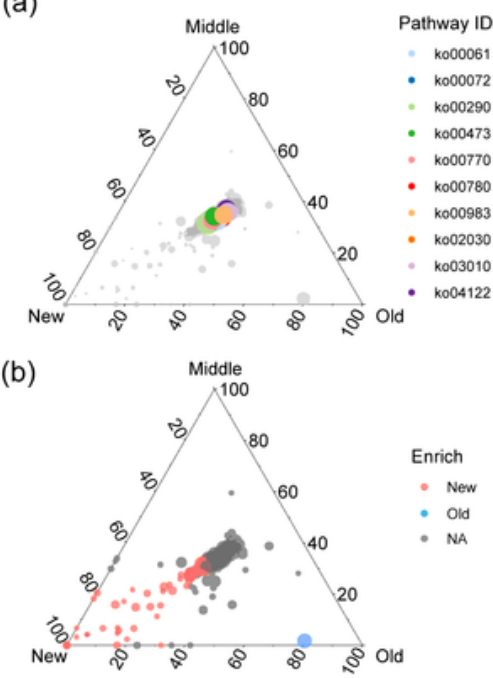

Old vs New

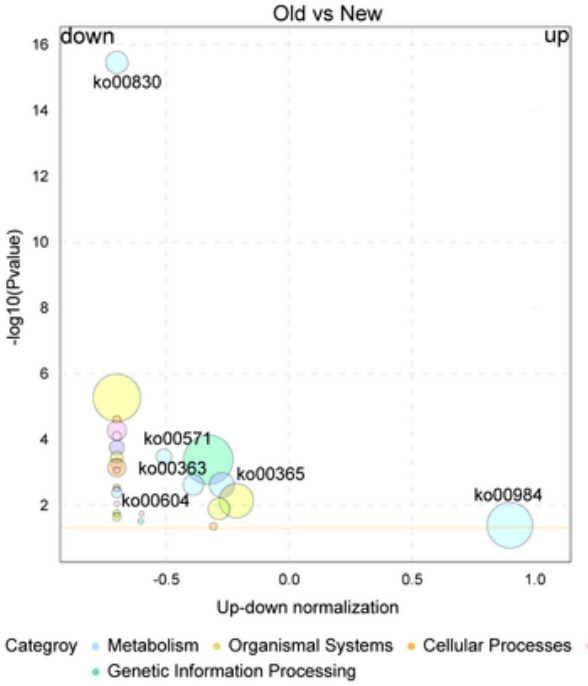

Top 20

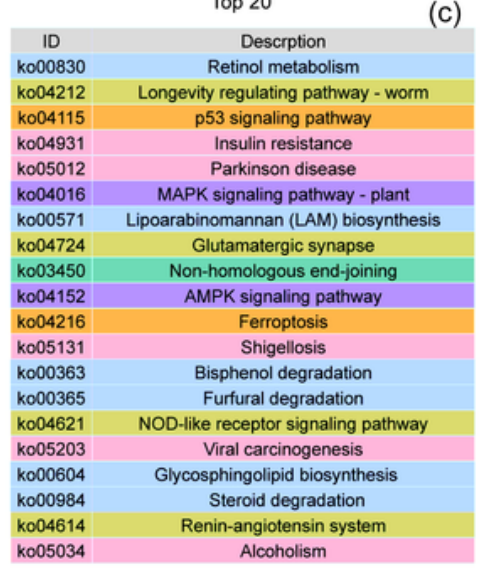

Steroid degradation ko00984

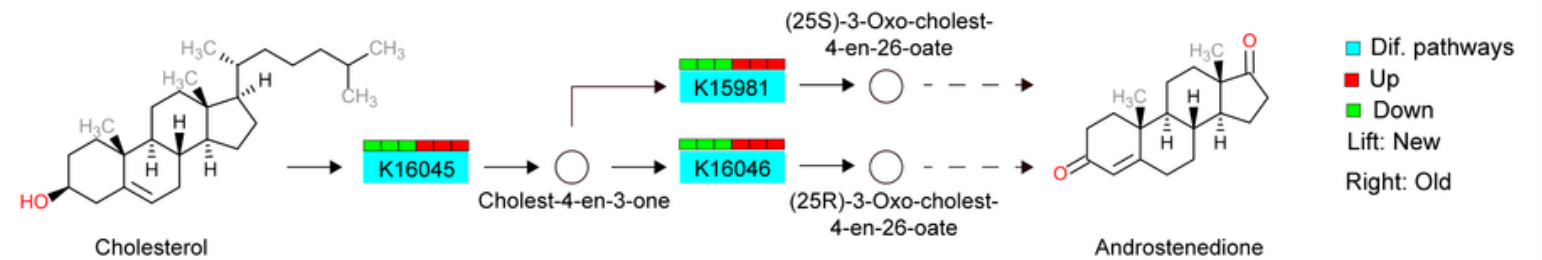

Furfural degradation ko00365

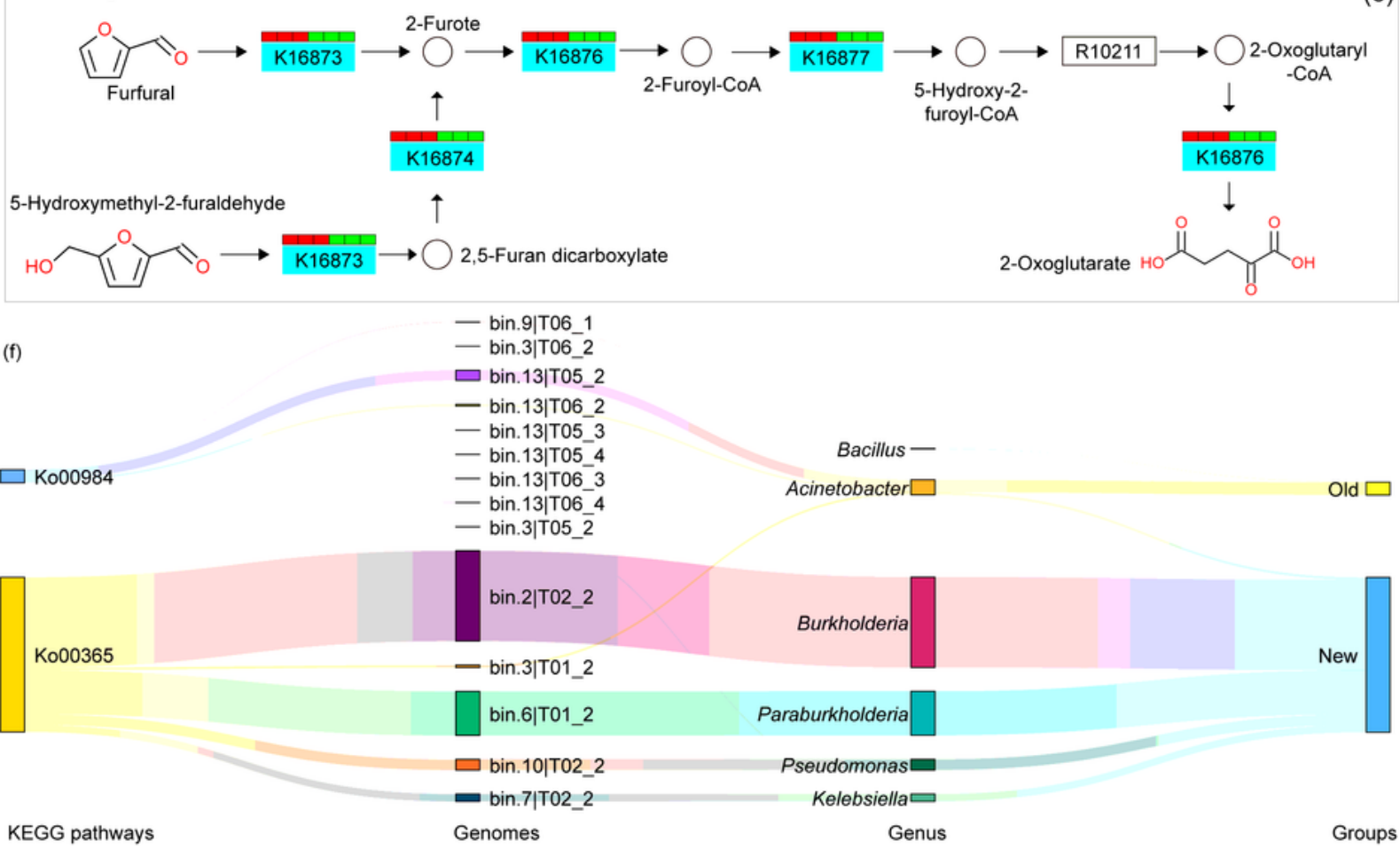

\section{Figure 4}

Effects of C. oleifera plantation age on degradation. (a). Variation in C. oleifera plantation ages with the distribution of the 10 most abundant KEGG pathways among soil metagenomes. (b). KEGG pathways significantly enriched in soil metagenomes from different plantation ages. (c). The twenty most abundant KEGG pathways that differentiated soil metagenomes from old and new C. oleifera forest plantations. (d). Differential enrichment of the steroid degradation pathway among soil metagenomes from different 
aged plantations. (e). Differential enrichment of the furfural degradation pathway among soil metagenomes from different aged plantations. (f). Genomes and genome annotation information for the pathways Ko00948 and Ko00365.

(a)
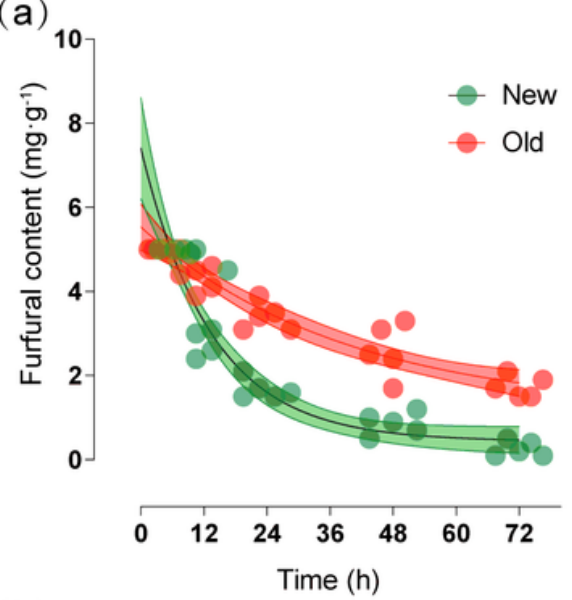

(b)

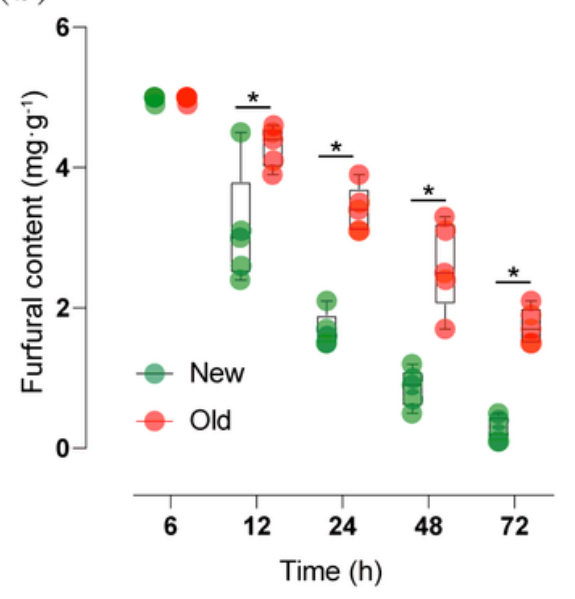

(c)
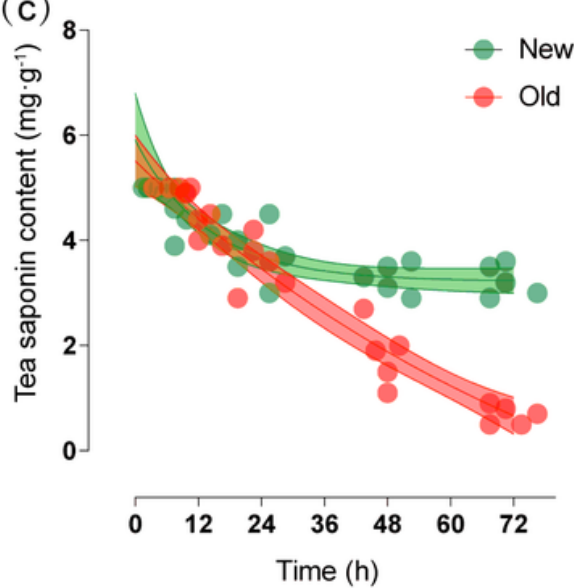

(d)

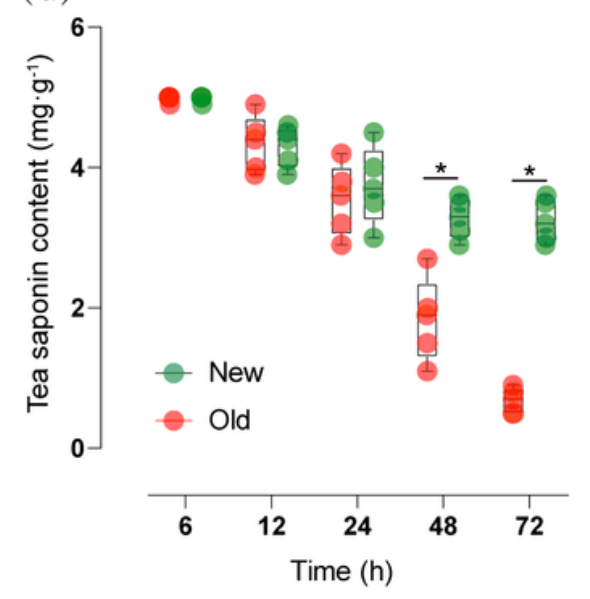

(e)
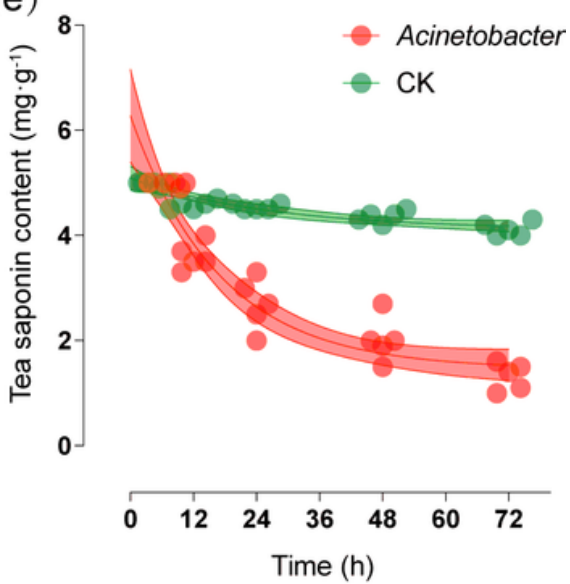

(f)

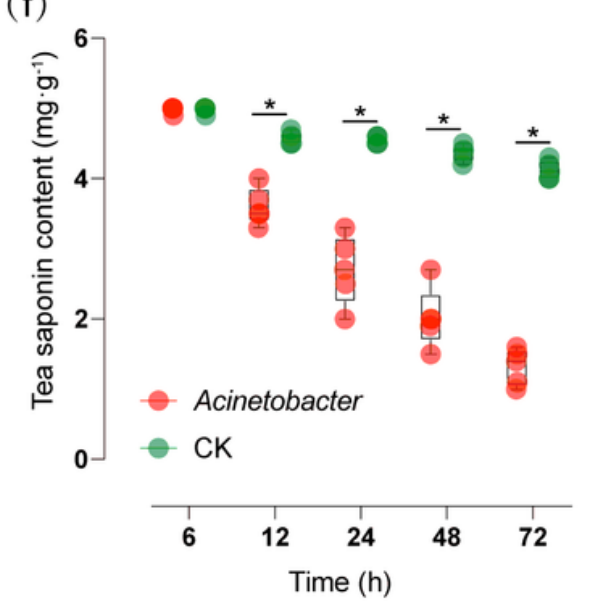

Figure 5

Degradation of furfural and tea saponin in soils of old and new C. oleifera plantations. 


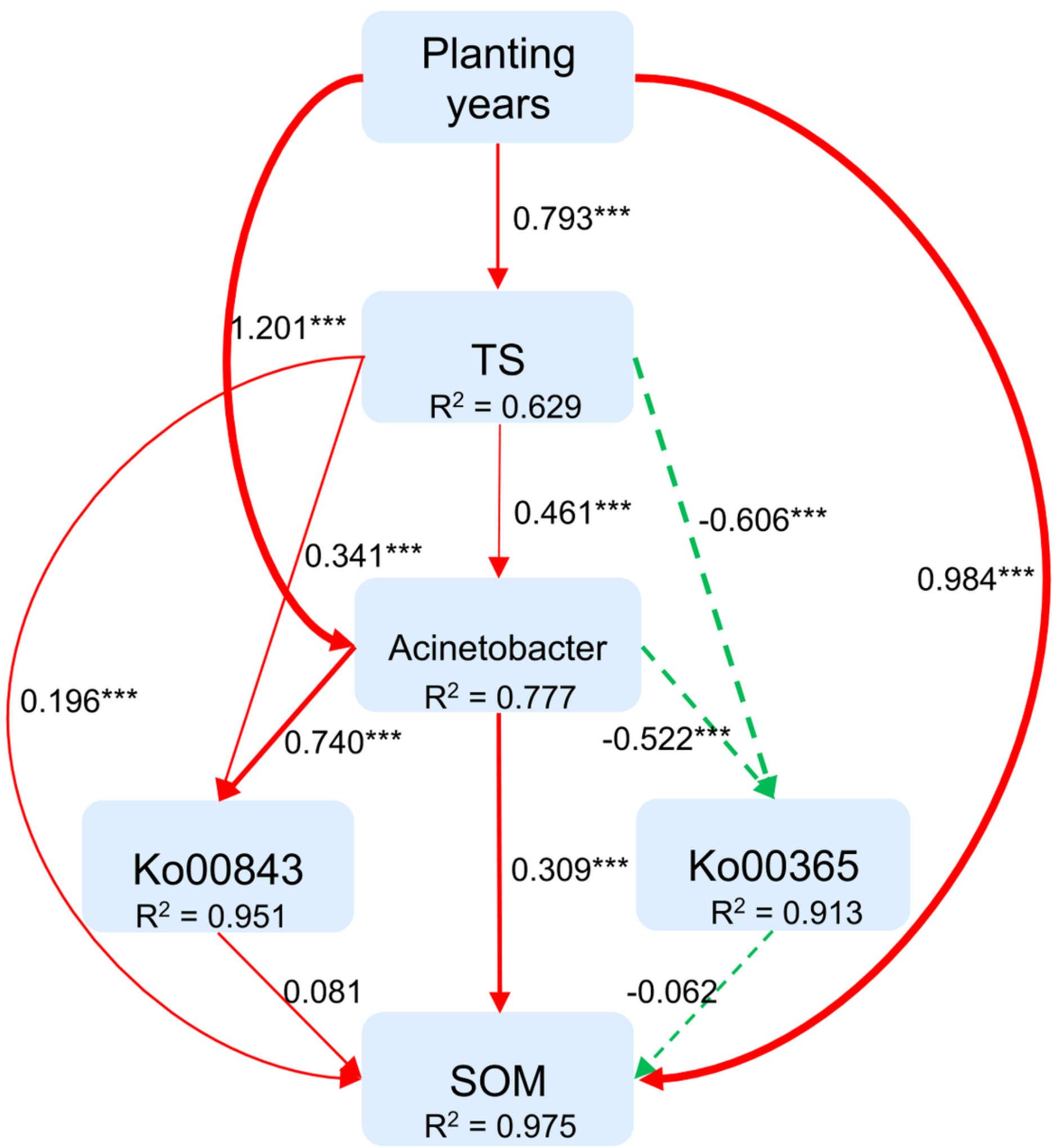

Figure 6

The structural equation model simulates the influence of PSMs on Ko00948 and Ko00365 pathways and SOM. (CFI = 0.992; GFI = 0.952; $\chi 2 / \mathrm{df}=3.862$ ). Continuous or dashed line indicates positive or negative relationships $\Downarrow$ respectively. Width of the arrow indicates strength of the effect. The digits besides the arrow are weight coefficients; R2 stands for size of the variable and RMSEA for root mean square error of approximation. ${ }^{\star \star \star}, \mathrm{P}<0.001$. 


\section{Supplementary Files}

This is a list of supplementary files associated with this preprint. Click to download.

- SupportingInformation.docx 